\section{Preproghrelin polymorphism Q90L (rs4684677) in gestational diabetes}

\author{
Polimorfismo Q90L (rs4684677) da preprogrelina \\ no diabetes gestacional
}

Rafaela Andrade Rocha', Henrique Ravanhol Frigeri', Izabella Castilhos Ribeiro dos Santos-Weiss', Rosângela Roginski Réa', Emanuel Maltempi de Souza', Fabiane Gomes de Moraes Rego', Geraldo Picheth'

$\mathrm{T}$ he preproghrelin gene (chromosome 3p26-p25) encodes two active peptides, ghrelin and obestatin. Both peptides are involved in the body energy homeostasis. Obestatin, a 23-amino-acid polypeptide, has the opposite effect to ghrelin, promoting reduced food intake and decreasing body weight ( 1 ). Low obestatin levels potentiate the insulin response to glucose, while high obestatin concentration inhibits insulin release (2). Several reports showed the association of single nucleotide polymorphisms (SNPs) of the preproghrelin gene with type 2 diabetes, obesity, insulin resistance, metabolic syndrome, anorexia nervosa, among other disorders (3). The association between the obesity pandemics and the increase in the occurrence of gestational diabetes suggested that variations in the preproghrelin gene could be good targets of a genetic marker for this disease. We investigated exon 3 of the preproghrelin gene, which encodes obestatin, for polymorphisms in gestational diabetes (GDM) in a case-controlled study. Healthy Euro-Brazilian pregnant women (control, $\mathrm{n}=165$ ) and gestational diabetic patients (GDM, n = 136) were classified according to American Diabetes Association criteria (4). Patients with overt diabetes were excluded. The Ethics Committee on Human Research of our institution approved this study. The obestatin encoding region was amplified by PCR (primers: F 5'-GGGCATGACCTCTGACATCT-3' and R 5'-GAAACCGAGCAAACCCAGT-3'; amplicon 19lbp) and polymorphisms were screened by PCR-SSCP. All different electrophoretic patterns and 15\% of other samples had their amplicons sequenced (BigDye, 3500 XL, Applied Biosystems). The SNP was identified and aligned using CodonCode Alligner v.4.1.1 (CodonCode Corporation) and the web sites BlastSNP and Reference SNP. The only polymorphism (SNP) identified was Q90L (rs4684677), a missense one (glutamine to leucine). We found no differences regarding genotype or allele frequencies for the SNP Q90L in the studied population. Also, the SNP was not associated with body mass index or fasting glucose levels (regression analysis, data not shown) in either group (Table 1). The rare allele $(T)$ frequency (11-12\%) observed was similar in Caucasians (10.8\%), and higher than in Asians ( 1\%) according to the HapMap (http://www.hapmap. org/). To our knowledge, this is the first report on this polymorphism in gestational diabetes patients. In conclusion, the SNP Q90L of the preproghrelin gene was not associated with gestational diabetes in the studied population.
Department of Clinical Analysis, and Department of Biochemistry and Molecular Biology, Universidade Federal do Parana (UFPR), Curitiba, PR, Brazil

Correspondence to: Geraldo Picheth Department of Clinical Analysis, Universidade Federal do Parana Curitiba, Parana, Brazil

Rua Prefeito Lothário Meissner, 632 80210-170 - Curitiba, PR, Brazil gpicheth@ufpr.br

Received on Dec/12/2013 Accepted on Dec/28/2013 
Table 1. Anthropometric parameters, clinical characteristics, genotype, and allele frequencies of Q90L polymorphism of preproghrelin gene in the absence (Control) or presence of gestational diabetes mellitus (GDM)

\begin{tabular}{lccc}
\hline Parameters & Control $\mathbf{~}=\mathbf{~ 1 6 5}$ & GDM $\mathbf{n}=\mathbf{1 3 6}$ & P \\
\hline Age, years & $24(20-29)$ & $33(29-39)$ & $<0.001$ \\
BMI, kg/m & $23.8(21.7-26.5)$ & $33.7(28.5-38.1)$ & $<0.001$ \\
FPG, mmol/L & $4.6(4.3 .0-4.8)$ & $5.5(5.1-6.7)$ & $<0.001$ \\
HbA1C, \% & - & $6.0(5.0-6.1)$ & - \\
\hline Genotyping (Q90L) & & & $\mathbf{0 . 7 6 3}^{*}$ \\
\hline A/A (QQ) & $128(77.6 \%)$ & $33(24.3 \%)$ & $1(0.7 \%)$ \\
A/T (QL) & $35(21.2 \%)$ & $12.9 \%[9-17 \%]$ & $0.696^{\star}$ \\
T/T (LL) & $2(1.2 \%)$ & & \\
T-allele [95\%Cl] & $11.8 \%[8-15 \%]$ & &
\end{tabular}

Values are medians (Interquartile Range) of non-normal distribution data, or $\mathrm{n}(\%)$. $95 \% \mathrm{Cl}, 95 \%$ confidence interval.

BMI: body mass index; FPG: fasting plasma glucose.

SNP Q90L; (Gln90Leu, rs4684677) are in Hardy-Weinberg equilibrium ( $P>0.05)$.

$\mathrm{P}$ value; Mann-Whitney $\mathrm{U}$ test or ${ }^{*}$ Chi-square test.

Acknowledgments: we are thankful to Dr. M. G. Yates for reading the manuscript, and to Valter A. Baura for his technical assistance. This project was supported by National Council for Scientific and Technological Development (CNPq) and the Araucaria Foundation.

Disclosure: no potential conflict of interest relevant to this article was reported.

\section{REFERENCES}

1. Epelbaum J, Bedjaoui N, Dardennes R, Feng DD, Gardette $\mathrm{R}$, Grouselle $\mathrm{D}$, et al. Role of the ghrelin/obestatin balance in the regulation of neuroendocrine circuits controlling body composition and energy homeostasis. Mol Cell Endocrinol. 2010;314(2):244-7.

2. Egido EM, Hernandez R, Marco J, Silvestre RA. Effect of obestatin on insulin, glucagon and somatostatin secretion in the perfused rat pancreas. Regul Pept. 2009;152(1-3):61-6.

3. Cheng KC, LiYX, Asakawa A, Inui A. The role of ghrelin in energy homeostasis and its potential clinical relevance (Review). Int $\mathrm{J}$ Mol Med. 2010;26(6):771-8.

4. American Diabetes Association. Diagnosis and classification of diabetes mellitus. Diabetes Care. 2013;36(Suppl 1):S67-74. 